\title{
CHARGE ASYMMETRY IN THE LEPTON PAIR DECAY OF POLARIZED RESONANCES ${ }^{\text {is }}$
}

\author{
Yukio TOMOZAWA \\ Randall Laboratory of Physics, University of Michigan, \\ Ann Arbor, MI 48109, USA
}

Received 3 October 1978

The charge asymmetry in the lepton pair of a polarized $\Upsilon$ (or any other heavy resonances) decay is computed. It provides a good test of the Weinberg-Salam model.

The study [e.g. 1] of the weak neutral current interaction seems to indicate that the standard WeinbergSalam (W-S) model [2] is consistent with various neutrino-induced reactions and the Novosivirisk experiment on the $\mathrm{Bi}$ atom [3], although the Oxford and Seattle experiment [4] on the Bi atom may contradict the W-S model. Recent asymmetry measurements of polarized electron deuteron scattering [5] seems to be best described by the WS model. However, lack of precision in the $y$-value measurement does not allow a decisive conclusion. In this article, it is shown that charge asymmetry in the decay, (polarized $\Upsilon \rightarrow \underset{\mu^{+}}{\mathrm{e}_{\mu}^{+}} \mathrm{e}^{-}$), is a sensitive test of the WS model. Of course, the asymmetry test in $\mathrm{e}^{+} \mathrm{e}^{-} \rightarrow \mu^{+} \mu^{-}$had been proposed since the early days of the PEP design [6]. Nevertheless, it is important to look at lepton pair data in the resonance regions, since (a) it has a smaller background and (b) it probes the property of the heavy quarks directly.

We assume that $\Upsilon$ or any other higher resonance expected to be observed in the PETRA and PEP experiments are polarized in the beam direction. This is what happened with the $\psi / \mathrm{J}$ resonance produced by SPEAR.

The relevant neutral current interaction in the W-S model is given by

\footnotetext{
Work supported in part by the U.S. Department of Energy.
}

$$
\begin{aligned}
\mathcal{L} & =\mathrm{i} e A_{\mu}\left(-\overline{\mathrm{e}} \gamma_{\mu} \mathrm{e}+\frac{2}{3} \overline{\mathrm{t}} \gamma_{\mu} \mathrm{t}-\frac{1}{3} \overline{\mathrm{b}} \gamma_{\mu} \mathrm{b}\right) \\
& +\frac{\mathrm{i} g Z_{\mu}^{0}}{4 \cos \theta_{\mathrm{w}}}\left(-\overline{\mathrm{e}} \gamma_{\mu} \mathrm{e}\left(1-4 \sin ^{2} \theta_{\mathrm{w}}\right)-\overline{\mathrm{e}} \gamma_{\mu} \gamma_{5} \mathrm{e}\right. \\
& +\overline{\mathrm{t}} \gamma_{\mu} \mathrm{t}\left(1-\frac{8}{3} \sin ^{2} \theta_{\mathrm{w}}\right)+\overline{\mathrm{t}} \gamma_{\mu} \gamma_{5} \mathrm{t} \\
& \left.-\overline{\mathrm{b}} \gamma_{\mu} \mathrm{b}\left(1-\frac{4}{3} \sin ^{2} \theta_{\mathrm{w}}\right)-\overline{\mathrm{b}} \gamma_{\mu} \gamma_{5} \mathrm{~b}\right),
\end{aligned}
$$

where $t,(b)$ stands for the top (bottom) quark with charge $2 / 3(-1 / 3)$. We notice that

$$
\left\langle 0\left|\overline{\mathrm{q}} \gamma_{\mu} \gamma_{5} \mathrm{q}\right|^{3} \mathrm{~S}_{1} \mathrm{q} \overline{\mathrm{q}} \text { bound state }\right\rangle=0
$$

since the axial current is even under charge conjugation while the ${ }^{3} S_{1}$ state is odd. Thus only the vector part of the quark current contributes to the weak neutral current decay of the resonances.

The leptonic decay of a polarized $\Upsilon$, is computed by

$$
\begin{aligned}
& |M|^{2} \propto \sum_{\text {spin }}\left|\epsilon_{\mu}\left(\overline{\mathrm{u}}\left(\gamma_{\mu}+A \gamma_{\mu} \gamma_{5}\right) \mathrm{v}\right)\right|^{2} \\
& \quad=\epsilon_{\mu} \epsilon_{\nu}^{*} \operatorname{Tr}\left[\frac{-\mathrm{i} \gamma p_{-}}{2 m} \gamma_{\mu} \frac{\mathrm{i} \gamma p_{+}}{2 m} \gamma_{\nu}\right. \\
& \left.\quad+2 A \frac{-\mathrm{i} \gamma p}{2 m} \gamma_{\mu} \gamma_{5} \frac{\mathrm{i} \gamma p_{+}}{2 m} \gamma_{\nu}\right] \\
& \quad=\frac{1}{m^{2}} \epsilon_{\mu} \epsilon_{\nu}^{*}\left[\left(p_{-}\right)_{\mu}\left(p_{+}\right)_{\nu}+\left(p_{+}\right)_{\mu}\left(p_{-}\right)_{\nu}+\frac{1}{2} m_{\mathrm{v}}^{2} \delta_{\mu \nu}\right. \\
& \left.\quad-2 A \epsilon_{\mu \nu \alpha \beta}\left(p_{-}\right)_{\alpha}\left(p_{+}\right)_{\beta}\right],
\end{aligned}
$$

where $p_{-}\left(p_{+}\right)$is the momentum of the massless lepton (antilepton) and $\epsilon_{\mu}$ and $m_{\mathrm{v}}$ are the polarization vector 
Table 1

The integrated asymmetry of the lepton pair in a polarized resonance decay

\begin{tabular}{|c|c|c|c|c|}
\hline & \multicolumn{2}{|c|}{$\sin ^{2} \theta_{w}=0.25$} & \multicolumn{2}{|c|}{$\sin ^{2} \theta=0.2$} \\
\hline & b-quark & t-quark & b-quark & $\mathrm{t}$-quark \\
\hline$r$ & -0.012 & -0.0030 & -0.013 & -0.0042 \\
\hline "30 GeV" & -0.12 & -0.030 & -0.13 & -0.041 \\
\hline
\end{tabular}

and the mass of the resonance respectively. For polarization $\epsilon_{\mu}=(1 / \sqrt{2}, \mathrm{i} / \sqrt{2}, 0,0)$, we obtain

$|M|^{2} \propto \frac{m_{\mathrm{v}}^{2}}{4 m^{2}}\left(1+\cos ^{2} \theta+4 A \cos \theta\right) \equiv f(\theta)$,

where the $z$ axis is chosen to be the direction of the spin polarization and the polar angle of $p_{+}$is $\theta$. The integrated asymmetry is then given by

$B=\frac{\int_{0}^{\pi / 2} f(\theta) \sin \theta \mathrm{d} \theta-\int_{\pi / 2}^{\pi} f(\theta) \sin \theta \mathrm{d} \theta}{\int_{0}^{\pi} f(\theta) \sin \theta \mathrm{d} \theta}=\frac{3}{2} A$.

The ratio of the axial vector and vector coupling, $A$, of the lepton pair is estimated from eq. (1),

$A=\frac{\left(G_{\pi} / 2 \sqrt{2}\right)\left(1-\frac{4}{3} \sin ^{2} \theta_{w}\right)}{-(4 \pi / 3) \alpha / m_{\mathrm{v}}^{2}} \quad$ for the $\mathrm{b}$ quark and

$A=\frac{\left(G_{\pi} / 2 \sqrt{2}\right)\left(1-\frac{8}{3} \sin ^{2} \theta_{\mathrm{W}}\right)}{-(8 \pi / 3) \alpha / m_{\mathrm{v}}^{2}}$ for the $\mathrm{t}$ quark.

Table 1 gives the numerical result for the integrated asymmetry, $B$, for various masses. For $\Upsilon$ or higher resonances, the observation of asymmetry is much easier if it is due to the bottom quark than if it is due to the top quark.

The author is indebted to Bob Levine for reading the manuscript.

\section{References}

[1] L.A. Abbott and R.M. Barnett, SLAC-PUB-2136 (1978); J.J. Sakurai, UCLA/78/TEP/9 (1978), and other references therein.

[2] S. Weinberg, Phys. Rev. Lett. 19 (1967) 1264; A. Salam, in: Elementary particle physics: Relativistic groups and analyticity, ed. N. Svartholm (Almquist and Wiksell, Stockholm, 1968) p. 367.

[3] L.M. Barkov and M.S. Zolotoryov, Pis'ma Zh. Eksp. Teor. Fiz. 27 (1978) 379 [JETP Lett., to be published].

[4] P.E. Baird et al., Phys. Rev. Lett. 39 (1977) 798;

L.L. Lewis et al., Phys. Rev. Lett. 39 (1977) 795.

[5] C.Y. Prescott et al., SLAC-PUB-2148 (1978).

[6] PEP Summer Study, PEP-137 (1974);

see also R. Gustafson et al. Fermilab proposal (1978). 